\title{
Aplicação de uma Metodologia para a Análise da Sensibilidade do Modelo Dinâmico para uma Tubulação-Separador sob Golfadas
}

A. SAUSEN 1 , P.S. SAUSEN², Departamento de Física, Estatística e Matemática, DeFEM, Departamento de Tecnologia, DeTec, Universidade Regional do Noroeste do Estado do Rio Grande do Sul, UNIJUÍ, 98700-000, Ijuí, RS, Brasil.

\begin{abstract}
Resumo. Neste artigo é apresentada a aplicação de uma metodologia para a análise da sensibilidade do modelo dinâmico para um sistema tubulação-separador sob regime de fluxo com golfadas, que é formado por um sistema de 5 (cinco) Equações Diferenciais Ordinárias (EDOs), não-lineares, acopladas, 6 (seis) parâmetros de sintonia e mais de 40 (quarenta) equações internas, geométricas e de transporte. Esta análise tem como objetivo verificar o efeito das variações dos parâmetros de sintonia sobre os resultados obtidos pela aplicação do modelo. A partir da análise dos resultados das simulações verificou-se que o modelo é altamente sensível a variação dos seus parâmetros de sintonia.
\end{abstract}

Palavras-chave. Golfadas, modelagem matemática, análise da sensibilidade.

\section{Introdução}

A golfada é considerada uma das mais preocupantes instabilidades em indústrias de produção de petróleo. Este tipo de escoamento caracteriza-se por fluxo severo e irregular, com ondas de líquido e gás através da seção transversal da linha de fluxo (i.e., tubulações), ocasionando oscilações de pressão e vazão na tubulação e nos equipamentos do processo $[6,10]$.

É importante destacar que a propagação da golfada gera diversos problemas indesejados na produção de petróleo, como por exemplo: alterações de períodos com nenhuma produção de líquido, seguido de períodos com grande produção de líquido dentro do separador, o que pode ocasionar redução na capacidade de produção, ineficiência da separação dos componentes da mistura, problemas nos trens de compressão e até inundações; também provoca desgaste, corrosão e danos nos equipamentos do processo $[2,3,4,8,9]$.

Por todos estes motivos, são necessárias estratégias que evitem a golfada na tubulação ou minimizem a mesma nas unidades de separação, de modo que seus efeitos não se propaguem para os equipamentos a jusante do processo. Em pesquisas recentes têm sido apontado que uma solução promissora para solucionar o problema da golfada é a utilização de estratégias de controle com realimentação $[1,2,3,6$, $8,9,10]$. Ressalta-se que esta metodologia é eficiente e econômica, pois é utilizado

\footnotetext{
1airam@unijui.edu.br.

${ }^{2}$ sausen@unijui.edu.br.
} 
um número reduzido de equipamentos na plataforma e na tubulação, há um menor desgaste dos equipamentos que compõem o processo, bem como menor custo com instalação e manutenção, e consequentemente é possível melhorar a regularidade e o desempenho do processo, aumentar a confiabilidade operacional da planta e, o mais importante, acelerar e aumentar a produção de petróleo.

Uma alternativa à aplicação de estratégias de controle com realimentação consiste em fazer uso de um modelo matemático que descreva adequadamente o processo. Em [6, 7] é apresentado um modelo matemático para um sistema tubulaçãoseparador sob regime de fluxo com golfadas através do acoplamento de duas situações: o modelo de uma tubulação sob golfadas $[9,10]$, e o modelo de um separador cilíndrico horizontal bifásico [6], conforme apresentado na Figura 1. Neste artigo, este modelo será denominado modelo de Sausen sendo formado por um sistema de 5 (cinco) Equações Diferenciais Ordinárias (EDOs), não-lineares, acopladas, com 5 (cinco) variáveis de estado, 6 (seis) parâmetros de sintonia (i.e., parâmetros empíricos) e mais de 40 (quarenta) equações internas, geométricas e de transporte.

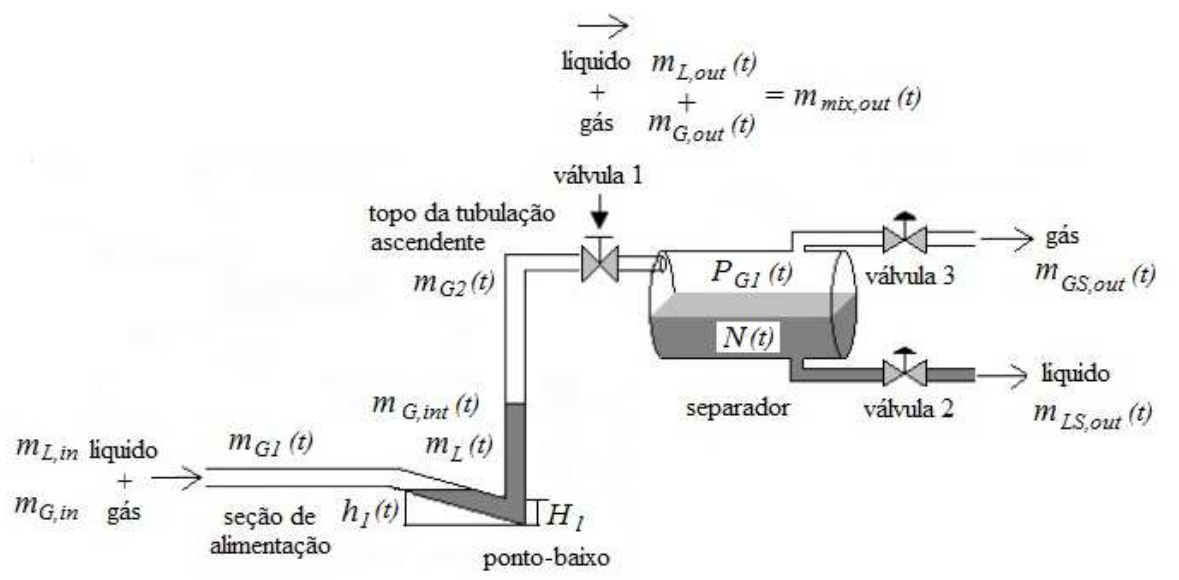

Figura 1: Ilustração de uma tubulação-separador com a golfada em formação.

Para a realização das simulações e aplicação de estratégias de controle com realimentação no modelo de Sausen, primeiramente, é necessário efetuar a sintonia do mesmo, isto é, o cálculo dos seus parâmetros empíricos. Para este procedimento devem ser utilizados dados de um estudo de caso, que podem ser obtidos a partir de uma planta experimental, de um simulador comercial, ou de um processo real. Os dados do estudo de caso considerados neste trabalho são obtidos dos estudos realizados por Storkaas et al. [8, 9, 10].

Após a realização da sintonia do modelo de Sausen $[6,7]$ destaca-se que é importante verificar o quanto as principais variáveis do modelo, tais como, fluxos mássicos de líquido e de gás, pressões na tubulação e no separador, entre outras, variam de comportamento considerando uma variação nos seus parâmetros de sintonia. Esta análise, denominada análise da sensibilidade, é uma ferramenta importante na construção de modelos matemáticos, além disto, proporciona uma melhor compreensão do comportamento dinâmico do sistema, para posteriormente aplicação de estratégias de controle com realimentação.

Portanto, o principal objetivo deste artigo é realizar a análise da sensibilidade no modelo de Sausen e verificar qual o parâmetro de sintonia que exerce maior 
influência nas principais variáveis do modelo. O presente artigo está organizado como segue. Na Seção 2. são descritas as equações do modelo de Sausen. Na Seção 3. é realizada a análise da sensibilidade do modelo de Sausen. Na Seção 4. são apresentados os resultados das simulações e sua análise. E por fim, na Seção 5. são apresentadas as conclusões.

\section{O Modelo}

Nesta seção são apresentadas as principais equações do modelo de Sausen $[6,7]$ baseadas nas equações de conservação de massa do sistema. A seguir, de acordo com a ilustração apresentada na Figura 1, as equações (2.1)-(2.3) descrevem a dinâmica da tubulação, e as equações (2.4)-(2.5) descrevem a dinâmica do separador

$$
\begin{gathered}
\dot{m}_{L}(t)=m_{L, \text { in }}-m_{L, \text { out }}(t) \\
\dot{m}_{G 1}(t)=m_{G, \text { in }}-m_{\text {Gint }}(t) \\
\dot{m}_{G 2}(t)=m_{G i n t}(t)-m_{G, \text { out }}(t) \\
\dot{N}(t)=\frac{\sqrt{r_{s}^{2}-\left(r_{s}-N(t)\right)^{2}}}{2 C \rho_{L} N(t)\left[3 r_{s}-2 N(t)\right]}\left[m_{L, \text { out }}(t)-m_{L S, \text { out }}(t)\right] \\
\dot{P}_{G 1}(t)=\frac{\left\{\rho_{L} \Phi\left[m_{G, \text { out }}(t)-m_{G S, \text { out }}(t)\right]+P_{G 1}(t)\left[m_{L, \text { out }}(t)-m_{L S, \text { out }}(t)\right]\right\}}{\rho_{L}\left[V_{S}-V_{L S}(t)\right]},
\end{gathered}
$$

onde $m_{L}(t)$ é a massa de líquido no ponto-baixo da tubulação, em $k g ; m_{G 1}(t)$ é a massa de gás na seção de alimentação da tubulação, em $k g ; m_{G 2}(t)$ é a massa de gás no topo da tubulação ascendente, em $k g ; N(t)$ é o nível de líquido dentro do separador, em $m ; P_{G 1}(t)$ é a pressão do gás dentro do separador, em $N / m^{2} ; \dot{m}_{L}(t)$, $\dot{m}_{G 1}(t), \dot{m}_{G 2}(t), \dot{N}(t)$, e $\dot{P}_{G 1}(t)$ são respectivamente suas derivadas em relação ao tempo; $m_{L, \text { in }}$ e $m_{G, \text { in }}$ são os fluxos mássicos de líquido e gás que entram na seção de alimentação da tubulação, considerados constantes, em $\mathrm{kg} / \mathrm{s} ; m_{L, \text { out }}(t)$ e $m_{G \text {,out }}(t)$ são os fluxos mássicos de líquido e gás que saem através da válvula 1 no topo da tubulação ascendente e por conseguinte entram no separador, em $\mathrm{kg} / \mathrm{s} ; m_{\text {Gint }}(t)$ é o fluxo mássico de gás interno na tubulação que flui da seção de alimentação para o topo da tubulação ascendente, em $\mathrm{kg} / \mathrm{s} ; m_{L S, \text { out }}(t)$ é o fluxo mássico de líquido que sai através da válvula 2 do separador, em $\mathrm{kg} / \mathrm{s} ; m_{G S, \text { out }}(t)$ é o fluxo mássico de gás que sai através da válvula 3 do separador, em $\mathrm{kg} / \mathrm{s} ; r_{\mathrm{s}}$ é o raio do separador, em $m ; C$ é o comprimento do separador, em $m ; \rho_{L}$ é a densidade de líquido no sistema, em $\mathrm{kg} / \mathrm{m}^{3} ; V_{S}$ é o volume total do separador, em $\mathrm{m}^{3} ; V_{L S}(t)$ é o volume de líquido no separador, em $m^{3} ; \Phi=\frac{R T}{M_{G}}$ é uma constante composta por $R$ constante universal do gases ideais $\left(8314 \frac{J}{K . k m o l}\right), T$ temperatura no sistema, em $K$; e $M_{G}$ peso molecular do gás, em $\mathrm{kg} / \mathrm{kmol}$.

O fluxo mássico da mistura $m_{m i x, o u t}(t)$ que sai da tubulação para o separador é determinado através da equação simplificada da válvula 1 no topo da tubulação ascendente, dada por

$$
m_{\text {mix }, \text { out }}(t)=z K_{1} \sqrt{\rho_{T}(t)\left(P_{2}(t)-P_{G 1}(t)\right)},
$$

onde $z$ é a abertura da válvula $(0-100 \%) ; K_{1}$ é um parâmetro de sintonia do modelo; $\rho_{T}(t)$ é a densidade na válvula, em $\mathrm{kg} / \mathrm{m}^{3} ; P_{2}(t)$ é a pressão no topo da tubulação ascendente, em $N / m^{2}$. 
O fluxo mássico de líquido e de gás que saem do separador são representados, respectivamente, pela equação da válvula 2 de líquido

$$
m_{L S, \text { out }}(t)=z_{L} K_{4} \sqrt{\rho_{L}\left[P_{G 1}(t)+g \rho_{L} N(t)-P_{O L 2}\right]},
$$

onde $z_{L}$ é a abertura da válvula $(0-100 \%) ; K_{4}$ é um parâmetro de sintonia do modelo; $g$ é a gravidade; $P_{O L 2}$ é a pressão a jusante da válvula de líquido, em $\mathrm{N} / \mathrm{m}^{2}$ e considerada constante; e pela equação da válvula 3 de gás, dada por

$$
m_{G S, \text { out }}(t)=z_{G} K_{5} \sqrt{\rho_{G}(t)\left[P_{G 1}(t)-P_{G 2}\right]},
$$

onde $z_{G}$ é a abertura da válvula $(0-100 \%) ; K_{5}$ é um parâmetro de sintonia do modelo; $\rho_{G}(t)$ é a densidade do gás, em $\mathrm{kg} / \mathrm{m}^{3} ; P_{G 2}$ é a pressão a jusante da válvula de gás, em $N / m^{2}$ e considerada constante.

Os fluxos mássicos de líquido e gás que entram na seção de alimentação da tubulação (i.e., $m_{L, i n}$ e $m_{G, i n}$ ) são considerados perturbações do processo e podem ser constantes, ou dependentes da pressão, neste trabalho são assumidos constantes. A seguir são apresentadas as equações que descrevem o deslocamento de gás e líquido dentro da tubulação.

\subsection{Deslocamento de gás na tubulação}

O deslocamento de gás ocorre através de uma relação entre o fluxo mássico de gás e a variação da pressão dentro da tubulação. Os dois principais parâmetros que determinam o deslocamento e a velocidade do gás no sistema são a variação da pressão $\Delta P(t)=P_{1}(t)-P_{2}(t)$ na tubulação, e a área livre no ponto-baixo, dada pelo nível de líquido relativo $f\left(h_{1}(t)\right)=\left(H_{1}-h_{1}(t)\right) / H_{1}$. Logo, a equação que representa o deslocamento de gás interno na tubulação, quando $h_{1}(t)<H_{1}$, é dada por

$$
v_{G 1}(t)=K_{2} f\left(h_{1}(t)\right) \sqrt{\frac{P_{1}(t)-P_{2}(t)-g \rho_{L} \alpha_{L}(t) H_{2}}{\rho_{G 1}(t)}},
$$

onde $K_{2}$ é um parâmetro de sintonia do modelo, $P_{1}(t)$ é a pressão na seção de alimentação da tubulação, em $N / m^{2} ; h_{1}(t)$ é o nível de líquido no ponto-baixo, em $m ; H_{1}$ é o diâmetro no ponto-baixo da tubulação, em $m ; \alpha_{L}(t)$ é a fração de líquido na tubulação ascendente; $H_{2}$ é a altura da tubulação ascendente, em $m$. Por outro lado, se $h_{1}(t) \geq H_{1}$ a velocidade de gás interna no ponto-baixo é igual a zero.

\subsection{Deslocamento de líquido na tubulação}

A distribuição de líquido ocorre através de uma equação de carregamento, ou seja, considera-se que o gás carrega o líquido pela tubulação ascendente, então modelase diretamente a fração de volume de líquido $\alpha_{L T}(t)$ que sai através da válvula 1 no topo da tubulação ascendente. É assumido que esta transição depende de um parâmetro $q(t)$, logo o carregamento de líquido é representado pela equação dada por

$$
\alpha_{L T}(t)=\alpha_{L T}^{*}(t)+\frac{q^{\varphi}(t)}{1+q^{\varphi}(t)}\left(\alpha_{L}(t)-\alpha_{L T}^{*}(t)\right),
$$

onde

$$
q(t)=\frac{K_{3} \rho_{G 1}(t) v_{G 1}^{2}(t)}{\rho_{L}-\rho_{G 1}(t)}
$$


e $K_{3}$ e $\varphi$ são parâmetros de sintonia do modelo. Os detalhes da modelagem matemática do modelo de Sausen podem ser encontrados em $[6,7]$ e suas equações internas, de transporte e geométricas podem ser encontradas em [6, 10].

\section{Análise da Sensibilidade}

Define-se a análise da sensibilidade de um sistema através da quantificação da variação do seu modelo dada uma mudança nos seus parâmetros de sintonia [5], ou seja, a sensibilidade é definida como uma relação entre um vetor de parâmetros do modelo

$$
\alpha=\left[\begin{array}{llll}
\alpha_{1} & \alpha_{2} & \ldots & \alpha_{r}
\end{array}\right]^{T}
$$

e um vetor de comportamento dinâmico do sistema

$$
\zeta=\left[\begin{array}{llll}
\zeta_{1} & \zeta_{2} & \ldots & \zeta_{s}
\end{array}\right]^{T}
$$

O vetor de parâmetros $\alpha$ pode ser decomposto como a combinação linear de outros dois vetores, isto é,

$$
\alpha=\alpha_{0}+\Delta \alpha,
$$

onde $\alpha_{0}$ são os valores nominais dos parâmetros e $\Delta \alpha$ são as variações em torno de $\alpha_{0}$. Do mesmo modo a decomposição pode ser realizada com o vetor que representa o comportamento dinâmico do sistema $\zeta$, isto é,

$$
\zeta=\zeta_{0}+\Delta \zeta
$$

onde $\zeta_{0}$ são os valores nominais do comportamento dinâmico do sistema e $\Delta \zeta$ são as variações em torno de $\zeta_{0}$.

Por conseguinte, é possível realizar a análise da sensibilidade partindo-se de um ponto de operação nominal $\left(\alpha_{0}, \zeta_{0}\right)$ e verificando-se quais alterações $\Delta \alpha$ nos parâmetros geram variações $\Delta \zeta$ no comportamento dinâmico do sistema.

O modelo de Sausen, descrito na Seção 2., possui 6 (seis) parâmetros de sintonia formando o seguinte vetor $\alpha$

$$
\alpha=\left[\begin{array}{llllll}
\varphi & K_{1} & K_{2} & K_{3} & K_{4} & K_{5}
\end{array}\right]^{T},
$$

cujos valores nominais são apresentados na Tabela 1 , o detalhamento do procedimento para a obtenção destes parâmetros pode ser encontrado em [6, 7]. E o vetor $\zeta(t)$ que caracteriza o comportamento dinâmico do sistema é dado por

$$
\zeta(t)=\left[\begin{array}{llllllll}
P_{1} & P_{2} & m_{L, \text { out }} & m_{G, \text { out }} & P_{G 1} & N & m_{L S, \text { out }} & m_{G S, \text { out }}
\end{array}\right]^{T} .
$$

Tabela 1: Parâmetros de sintonia para o modelo dinâmico de um sistema tubulaçãoseparador.

\begin{tabular}{|c|c|c|c|c|c|}
\hline$\varphi$ & $K_{1}$ & $K_{2}$ & $K_{3}$ & $K_{4}$ & $K_{5}$ \\
\hline 2,55 & 0,005 & 0,8619 & 1,2039 & 0,002 & 0,0003 \\
\hline
\end{tabular}

A análise da sensibilidade é realizada considerando os vetores apresentados nas equações (3.1) e (3.2), aplicando a seguinte metodologia: inicialmente é escolhido um dos parâmetros de sintonia do vetor $\alpha$ (e.g., $K_{1}$ ), então, altera-se sucessivamente 
o valor do mesmo em cada uma das simulações considerando-se todos os demais parâmetros (i.e., $\varphi, K_{2}, K_{3}, K_{4}$ e $K_{5}$ ) com seus valores nominais (i.e., de referência), e finalmente são analisados os resultados destas simulações verificando quais alterações ocorrem nos parâmetros que representam o comportamento dinâmico do sistema (i.e, vetor $\zeta(t)$ ). As simulações são realizadas na ferramenta matemática MATLAB, e para todos os casos simulados considera-se primeiramente a abertura da válvula 1 no topo da tubulação ascendente igual a $z=12 \%$ (i.e., regime de fluxo sem golfadas), e posteriormente igual a $z=20 \%$ (i.e., regime de fluxo com golfadas). Mais detalhes referente ao comportamento dinâmico do modelo, em relação a abertura da válvula 1 , podem ser obtidos em $[6,7,10]$.

\section{Resultados e Análise das Simulações}

Nesta seção são apresentados os resultados das simulações e sua análise considerando a realização da análise da sensibilidade do modelo de Sausen. Observa-se que o vetor $\zeta(t)$ é composto por 8 (oito) variáveis do modelo, porém aqui para efeito de simplificação e restrição de espaço são apresentados apenas os resultados das simulações para as variáveis $P_{1}(t)$ e $P_{2}(t)$, que representam, respectivamente, as pressões na seção de alimentação e no topo da tubulação ascendente, sendo responsáveis pela formação da golfada no sistema; e $N(t)$ e $P_{G 1}(t)$ que descrevem, respectivamente, a dinâmica do nível de líquido e da pressão do gás no separador. Os demais resultados, para as outras variáveis que compõem o vetor $\zeta(t)$, podem ser encontrados em [6].

Para a análise da sensibilidade do modelo de Sausen, inicialmente, variou-se o parâmetro de sintonia $K_{1}$ na equação da válvula 1 no topo da tubulação ascendente, equação (2.6). Na Figura 2 (a) é apresentado o resultado das simulações para a variável do modelo $P_{1}(t)$ e na Figura 2 (b) é apresentado o resultado das simulações para a variável $P_{2}(t)$ considerando a abertura da válvula 1 no topo da tubulação ascendente igual a $12 \%$. Nas Figuras 3 (a) e (b) são considerados as mesmas variáveis do modelo, respectivamente $P_{1}(t)$ e $P_{2}(t)$, mas a abertura da válvula 1 é igual a $20 \%$. As curvas principais (i.e., as curvas centrais e sólidas) correspondem aos resultados das simulações com o valor nominal $K_{1}=0,005$. Observa-se, conforme apresentado na Figura 2 (a) e (b), que as demais curvas apresentam características da curva principal com oscilações amortecidas apenas quando o novo $K_{1}$ está muito próximo de 0,005 (valor nominal), ou seja, em $K_{1}=0,004$ e $K_{1}=0,006$. Para valores superiores, como por exemplo, $K_{1}=0,007$ e $K_{1}=0,008$, as curvas apresentam oscilações periódicas com características diferentes da curva principal. Conforme apresentado na Figura 3 (a) e (b), para $z=20 \%$, as demais curvas apresentam características da curva principal com oscilações periódicas apenas quando o novo $K_{1}$ está muito próximo de 0,005 , ou seja, em $K_{1}=0,006$. Para $K_{1}=0,004$ observou-se que a curva possui oscilações amortecidas.

O segundo parâmetro a ser analisado é o $K_{2}$, na equação da velocidade do gás na tubulação, equação (2.9). Observa-se, partindo de alterações do valor nominal $K_{2}=0,8619$, que as demais curvas simuladas não possuem as características da curva principal (i.e., curva central e sólida) com oscilações amortecidas para $z=12 \%$ e com oscilações periódicas para $z=20 \%$. As curvas dos resultados das simulações para $K_{2}$ diferente do valor nominal são amortecidas bruscamente conforme pode ser observado nas Figuras 4 (a) e (b) para $z=12 \%$ e nas Figuras 5 (a) e (b) para $z=20 \%$. 

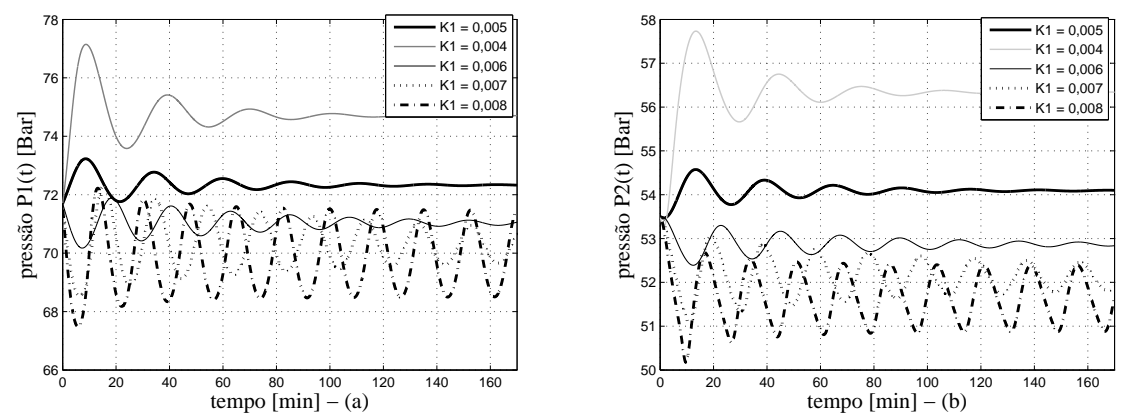

Figura 2: (a) Variações da pressão $P_{1}(t)$ e (b) variações da pressão $P_{2}(t)$, considerando alterações no parâmetro de ajuste $K_{1}$ e $z=12 \%$.
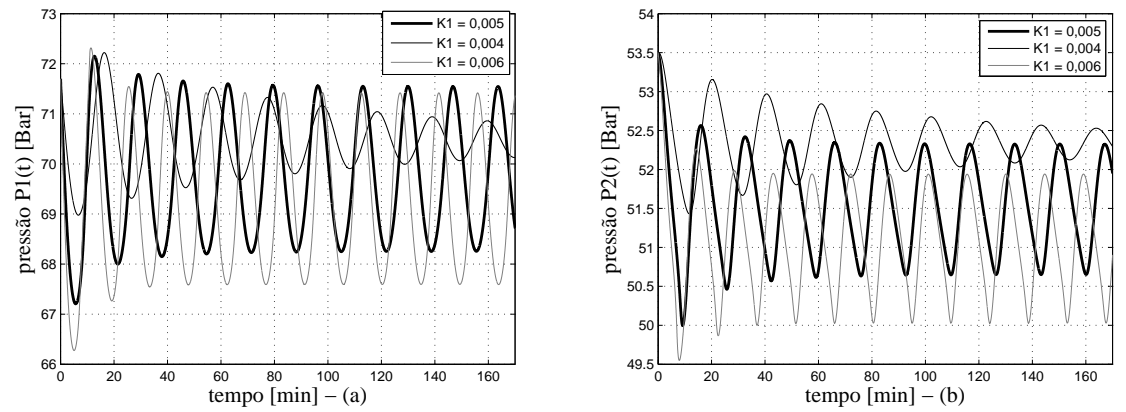

Figura 3: (a) Variações da pressão $P_{1}(t)$ e (b) variações da pressão $P_{2}(t)$, considerando alterações no parâmetro de ajuste $K_{1}$ e $z=20 \%$.
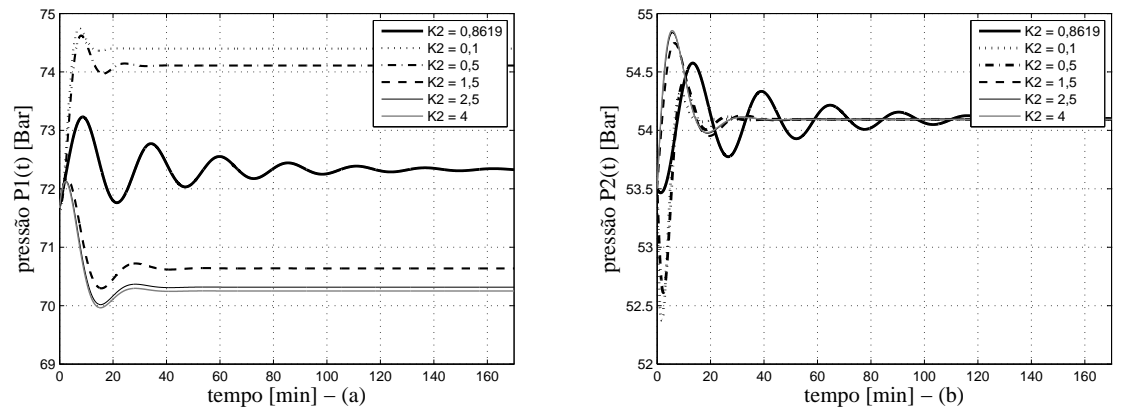

Figura 4: (a) Variações da pressão $P_{1}(t)$ e (b) variações da pressão $P_{2}(t)$, considerando alterações no parâmetro de ajuste $K_{2}$ e $z=12 \%$.

O terceiro parâmetro a ser avaliado é o $\varphi$, na equação de carregamento de líquido na tubulação, equação (2.10), observa-se conforme as Figuras 6 (a) e (b), para $z=12 \%$, partindo de alterações do valor nominal $\varphi=2,55$, que as curvas apresentam características da curva principal (i.e., curva central e sólida) com oscilações amortecidas, quando o novo $\varphi$ está próximo de 2, 55 (valor nominal), por exemplo, em $\varphi=3$. Quando utiliza-se um valor que não está próximo do valor nominal, a curva apresenta oscilações periódicas, ou é amortecida rapidamente. Nas Figuras 7 (a) e (b), para $z=20 \%$, partindo do valor nominal $\varphi=2,55$, também verifica-se 

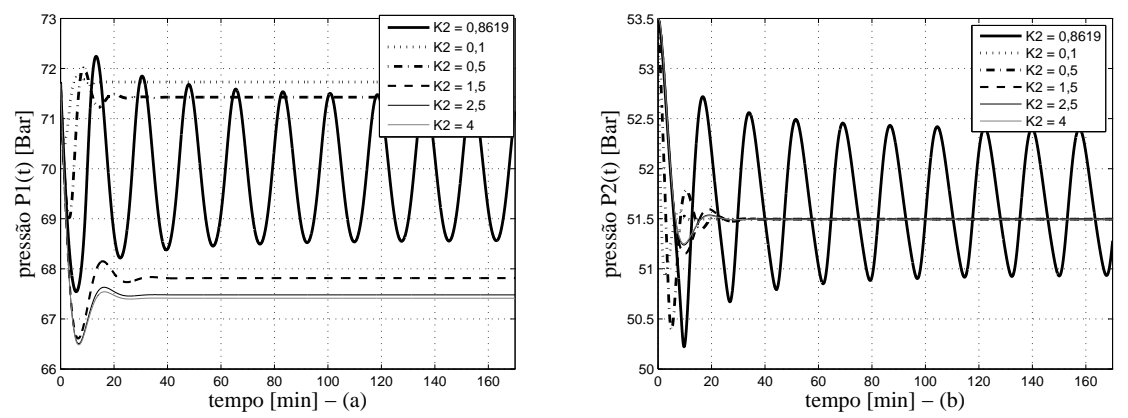

Figura 5: (a) Variações da pressão $P_{1}(t)$ e (b) variações da pressão $P_{2}(t)$, considerando alterações no parâmetro de ajuste $K_{2}$ e $z=20 \%$.

que as curvas possuem características da curva principal apenas para valores de $\varphi$ próximos ao valor nominal.

Com a variação do quarto parâmetro $K_{3}$, na equação de carregamento de líquido na tubulação, equação (2.10), observa-se para $z=12 \%$, partindo de alterações do valor nominal $K_{3}=1,2039$, que as curvas apresentam características da curva principal (i.e., curva central e sólida) com oscilações amortecidas quando o novo $K_{3}$ está próximo de 1,2039 (valor nominal), por exemplo, em $K_{3}=1,5$, conforme Figuras 8 (a) e (b), para os demais casos simulados as curvas são amortecidas bruscamente. Verifica-se para $z=20 \%$, partindo de alterações do valor nominal $K_{3}=1,2039$, que todas as curvas apresentam características diferentes da curva principal, segundo pode ser verificado nas Figuras 9 (a) e (b).
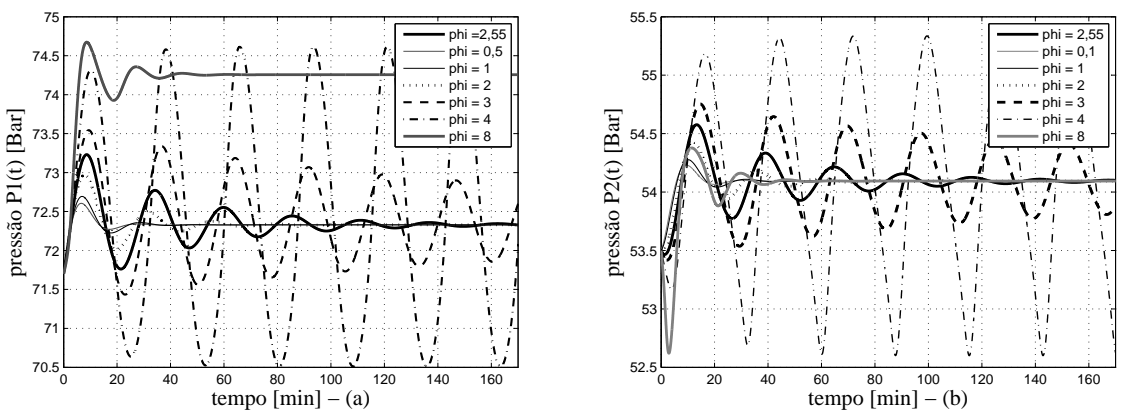

Figura 6: (a) Variações da pressão $P_{1}(t)$ e (b) variações da pressão $P_{2}(t)$, considerando alterações no parâmetro de ajuste $\varphi$ e $z=12 \%$.

Até então foram apresentados os resultados das simulações para a análise da sensibilidade dos parâmetros de sintonia da tubulação. A seguir são apresentados os resultados das simulações para a variação dos parâmetros de sintonia do separador. $\mathrm{Na}$ sequência, o quinto parâmetro avaliado é o $K_{4}$, na equação da válvula 2 de líquido do separador, equação (2.7). Verifica-se para $z=12 \%$ e $z=20 \%$, partindo de alterações do valor nominal de $K_{4}=0,002$ para $K_{4}=0,0015$, e então para $K_{4}=0,0025$, que houveram alterações na condição de equilíbrio do tanque, ou seja, o nível de líquido no tanque aumentou quando $K_{4}$ foi reduzido, e diminuiu quando $K_{4}$ foi aumentado, o mesmo ocorrendo para a pressão do gás, conforme 

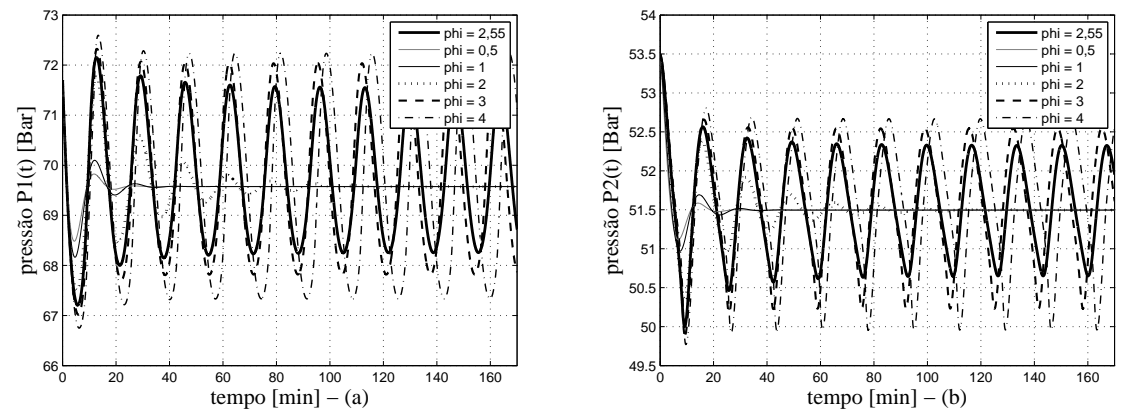

Figura 7: (a) Variações da pressão $P_{1}(t)$ e (b) variações da pressão $P_{2}(t)$, considerando alterações no parâmetro de ajuste $\varphi$ e $z=20 \%$.
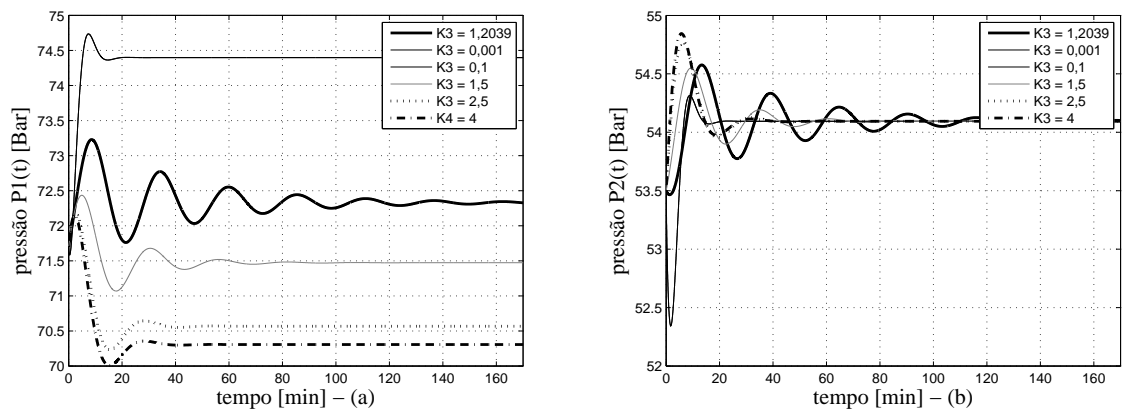

Figura 8: (a) Variações da pressão $P_{1}(t)$ e (b) variações da pressão $P_{2}(t)$, considerando alterações no parâmetro de ajuste $K_{3}$ e $z=12 \%$.
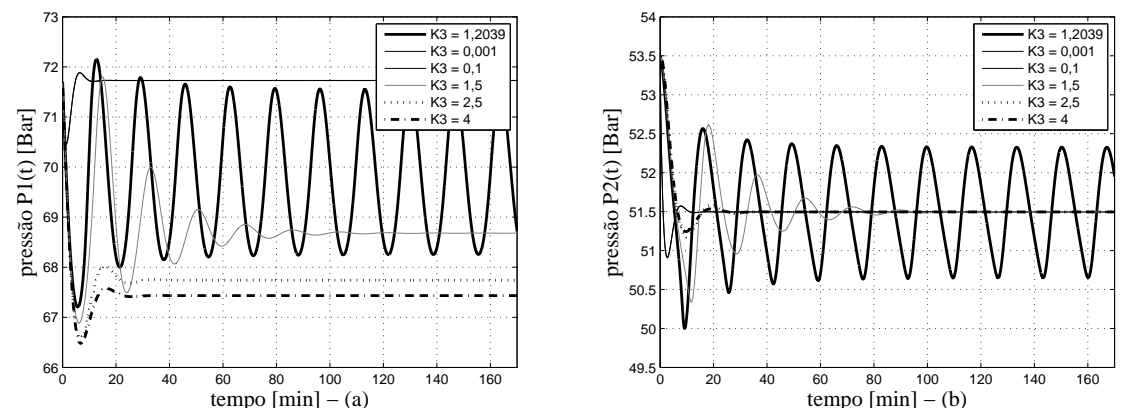

Figura 9: (a) Variações da pressão $P_{1}(t)$ e (b) variações da pressão $P_{2}(t)$, considerando alterações no parâmetro de ajuste $K_{3} \mathrm{e} z=20 \%$.

pode ser observado nas Figuras 10 (a) e (b) e nas Figuras 11 (a) e (b).

E por fim, variou-se o parâmetro $K_{5}$ na equação da válvula de gás do separador, equação (2.8). Observa-se para $z=12 \%$ e $z=20 \%$, partindo de alterações do valor nominal de $K_{5}=0,0003$ para $K_{5}=0,0002$ e então para $K_{5}=0,00035$, que houveram alterações na condição de equilíbrio do tanque, ou seja, o nível de líquido no tanque subiu quando $K_{5}$ foi aumentado, e diminuiu quando $K_{5}$ foi reduzido, o mesmo acontecendo para a pressão do gás dentro do vaso, como pode ser observado 

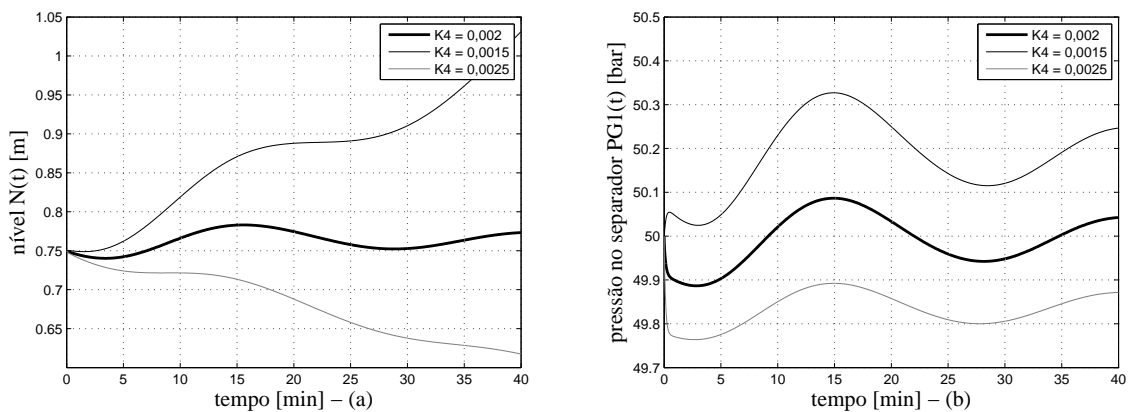

Figura 10: (a) Variações do nível de líquido $N(t)$ e (b) variações da pressão do gás $P_{G 1}(t)$, considerando alterações no parâmetro de ajuste $K_{4}$ e $z=12 \%$.
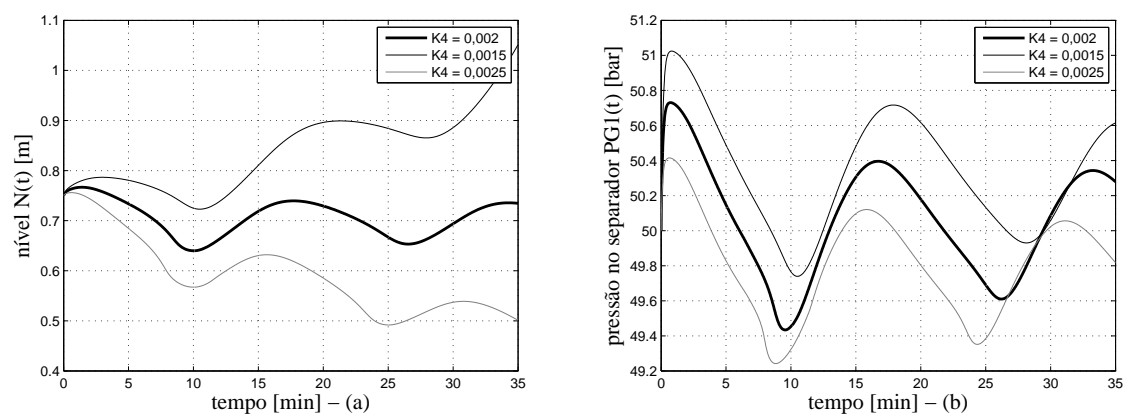

Figura 11: (a) Variações do nível de líquido $N(t)$ e (b) variações da pressão do gás $P_{G 1}(t)$ dentro do separador, considerando alterações no parâmetro de ajuste $K_{4}$ e $z=20 \%$.

nas Figuras 12 (a) e (b) e nas Figuras 13 (a) e (b).
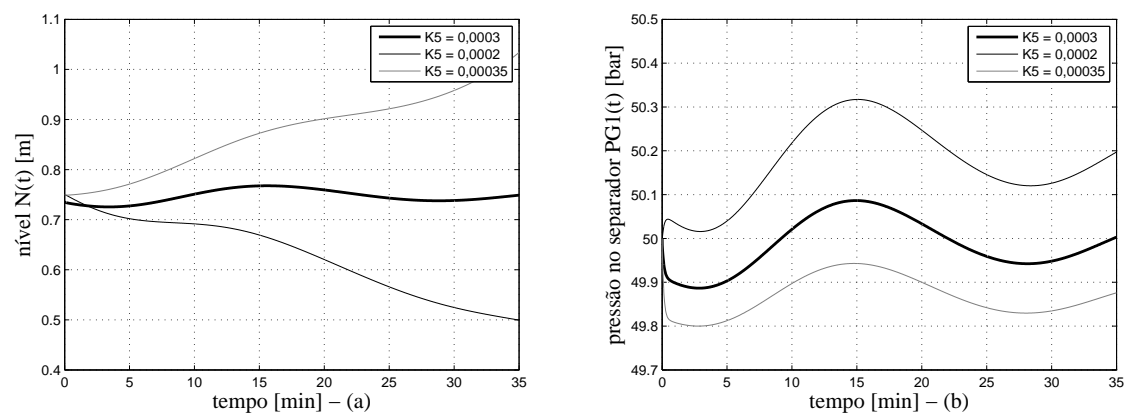

Figura 12: (a) Variações do nível de líquido $N(t)$ e (b) variações da pressão do gás $P_{G 1}(t)$, considerando alterações no parâmetro de ajuste $K_{5}$ e $z=12 \%$. 

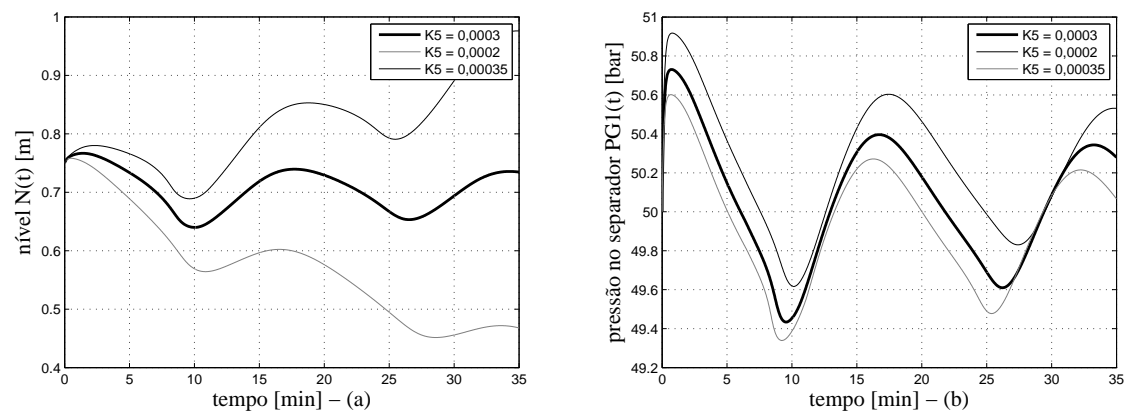

Figura 13: (a) Variações do nível de líquido $N(t)$ e (b) variações da pressão do gás $P_{G 1}(t)$ dentro do separador, considerando alterações no parâmetro de ajuste $K_{5}$ e $z=20 \%$.

\section{Conclusões}

Neste trabalho, a partir da análise dos resultados das simulações, conclui-se que o modelo aqui apresentado mostrou-se sensível as variações de todos os parâmetros de sintonia, sendo altamente sensível as alterações nos parâmetros $K_{2}$ na equação da velocidade do gás, e $K_{3}$ na equação de carregamento de líquido na tubulação ascendente.

Observa-se que os parâmetros de sintonia $K_{2}$ e $K_{3}$ influenciam diretamente na formação da golfada, pois compõem, respectivamente, as equações que descrevem o deslocamento de gás e de líquido na tubulação. Verifica-se que os resultados das simulações obtidos com valores diferentes dos seus respectivos valores nominais acarretaram mudanças significativas nos resultados das simulações das variáveis do modelo, $P_{1}(t)$ e $P_{2}(t)$, que geram o regime de fluxo com golfadas no sistema. Ou seja, as curvas com outros valores, diferentes dos valores nominais, não possuem características da curva principal (i.e., curva correspondente ao parâmetro de sintonia com valor nominal), pois não ocorreram oscilações decrescentes que caracterizam o regime de fluxo sem golfadas, ou oscilações periódicas que descrevem as golfadas no sistema, sendo que as curvas são amortecidas bruscamente.

A partir dos resultados das simulações pode-se afirmar que os parâmetros de sintonia do modelo de Sausen devem estar devidamente sintonizados para somente assim representarem coerentemente o regime de fluxo com golfadas, tanto na tubulação quanto no separador.

Abstract. This paper presents the application of a methodology for sensitivity analysis of the dynamic model for a pipeline-separator system, which is formed for 5 (five) Ordinary Differential Equations (ODEs) coupled nonlinear, 6 (six) tuning parameters and more than 40 (forty) internal, geometric and transport equations. This analysis aims to determine the effect of the variations of the tuning parameters on the results obtained by applying the dynamic model. From the analysis of simulation results verified that the dynamic model is highly sensitive to changes in its tuning parameters.

\section{Referências}

[1] A.M. Borges, M.A.A. de Meira, C.E.M. Vaz, Sistema de controle de golfadas, em "Rio Oil e Gas Expo and Conference 2004", Rio de Janeiro, 2004. 
[2] M.C.M. de Campos, L.A. Costa, A.E. Torres, D.C. Schmidt, Controle avançado dos níveis dos separadores de plataformas de produção, em " $1^{o}$ CICAP Congresso de Instrumentação, Controle e Automação da Petrobrás (I CICAP)", RJ, 2008.

[3] M.C.M. Campos, P. Laureiro, A.M. Borges Filho, Novas estratégias de controle para a plataforma de petróleo P-55, em "Rio Oil e Gas Expo and Conference 2006", Rio de Janeiro, 2006.

[4] M.J. Godhavn, F.P. Mehrdad, P. Fuchs, New slug control strategies, tuning rules and experimental results, Journal of Process Control, 15 (2005), 547-577.

[5] H.K. Khalil, "Nonlinear Systems", Prentice Hall, New Jersey, 2002.

[6] A. Sausen, "Modelagem Matemática de um Sistema Tubulação-Separador sob Regime de Fluxo com Golfadas e Controle de Nível Considerando um Algoritmo de Erro-Quadrático", Tese de Doutorado, Universidade Federal de Campina Grande-UFCG, Brasil, 2009.

[7] A. Sausen, P.R. Barros, Modelo dinâmico simplificado para um sistema encanamento-riser-separador sob regime de fluxo com golfadas, em "Seleta do Dincon 2008" (Meneguette Jr. e outros, eds.), TEMA - Tendências em Matemática Aplicada e Computacional, Vol. 9, No. 2, pp. 341-350, SBMAC, 2008.

[8] E. Storkaas, S. Skogestad, Controllability analysis of two-phase pipeline-riser systems at riser slugging conditions, Journal of Process Control, 15 (2006), $567-581$.

[9] E. Storkaas, S. Skogestad, J.M. Godhavn, Stabilization of severe slugging based on a low-dimensional nonlinear model, em "AIChE Annual Meeting", Paper 259e, Indianapolis, USA, 2002.

[10] E. Storkaas, "Stabilizing Control and Controllability: Control Solutions to Avoid Slug Flow in Pipeline-Riser Systems", Tese de Doutorado, Norwegian University of Science and Technology, Norwegian, 2005. 